\title{
A NOVEL APPROACH FOR CLUSTER HEAD SELECTION USING TRUST FUNCTION IN WSN
}

\author{
VIPUL NARAYAN*AND A.K. DANIEL ${ }^{\dagger}$
}

\begin{abstract}
The enhancement of new technology in the sensor network shows a significant result in every aspect of life such as military surveillance, hospitals, mining and hospitals etc. The nodes are scattered randomly in RoI (Region of Interest) and data is transmitted to Base Station (BS) using the multi-hop technique. The Wireless Sensor Network (WSN) become an important research field for challenging problems as energy consumption, efficient cluster head selection process, routing algorithm, network strength, packet loss, energy loss and so forth. The agenda in the paper is to enhance Residual Energy (RE) of nodes and network lifetime. The problem is solved by using an efficient clustering and Cluster Head $(\mathrm{CH})$ selection process. The cluster head selection is based on the maximum node residual energy and the minimum distance from the base station. The Proposed protocol worked in two stages. The new Threshold value $\mathrm{T}(\mathrm{H})$ is calculated for the cluster head selection process in the first stage. The data fusion method based on the trust function is used to get accurate data in the second stage. The energy model is utilized to reduce the excessive energy transmission inside the network. The Proposed protocol is compared with Stable Election Protocol and achieves $44 \%$ lifetime improvement, $59 \%$ stability improvement and $15 \%$ in survival rate respectively.
\end{abstract}

Key words: Clustering, Cluster Head Selection, Energy Efficiency, Wireless Sensor Network, Trust.

AMS subject classifications. 68M14

1. Introduction. Recent growth in the field of the mobile internet and sensor network technology has motivated the mindset of people towards new technology [1][2]. The Sensor Nodes (SN) are distributed to cover the network area and interact with the external environment using certain communication factors. The SN have limited battery power. The multiple SN are required to perform real-time tracking of objects in the network. In the twenty-one century, the WSN has played a significant impact on human lives. The evolution of science engineering and advanced technology in many disciplines improve sensor nodes technology and influences daily life nowadays. In year 1970s, the first generation sensor network was introduced. The SN follow point to point communication to transfer the data to BS. The next generation sensor network instead comprises less energy and operated independently with the cooperation of former nodes and collects data in the network. In the early 1990s, the third generation sensor network was introduced in the market with advance features and use a device manager and bus connection system to collect the information in the network. The fourth generation sensor networks have an advanced characteristic which performs a multi-hop approach and self-organizing features in the network for data transmission in the WSN [3][4]. The sensor network collects the information from various sources and sent to BS via single/multiple hop fashion. The sensor objects and observers play a significant role together for establishing communication via the communication link in the network. The multiple SN are deployed in the critical zone area after regular interval of time because data collection in these places are very difficult. The location of every SN in the network is not easily tracked. The GPS is used in some sensor nodes to know the exact location in the network. The sensor network has played an important role in the current scenario as deployement strategy,protocol design and green communication etc.[5]. The various applications are handled remotely and embedded therefore,requirements of sensor network in every place for the scientific and research purpose [6]. The modern sensor network technology is much more efficient than the traditional network. The node energy and environmental interruption affects the communication in the network. The

\footnotetext{
*Department of Computer Science and Engineering, Madan Mohan Malaviya University of Technology, Gorakhpur, India. (vipulupsainian2470@gmail.com).

${ }^{\dagger}$ Department of Computer Science and Engineering, Madan Mohan Malaviya University of Technology, Gorakhpur, India. (danielak@rediffmail.com).
} 
effective route optimization technique and utilization of resources in the network solve the problem which maximizes bandwidth utilization and maintain QoS in the WSN. The nodes are placed at static location in the hazardous region and sometimes unable to cover the network area due to several obstructions. The problem is solved by using energy efficient-coverage protocol and improves network lifetime. The RSSI is used to determine the current power of the signal. The complete coverage and optimum utilization of energy while transmitting the data in in the network become important research in the present scenario [7][8]. There are two types of transmission in the sensor network. The first is the actual transmission and another is the data fusion rate. In actual transmission the data is lost due to environment factors and data corruption. The data fusion rate helps in node computing, storage computing and reduce the consumption of energy inside the WSN. The nodes are randomly distributed in network and every time long distance node use more RE compared to shortest distance.

The paper proposed a protocol that uses new Threshold value for advanced nodes and normal nodes to prevent randonmness in the selection process of $\mathrm{CH}$. The new Threshold value consits of weighted energy and distance ratio to prevent $\mathrm{CH}(\mathrm{s})$ and low node failure. The data fusion method based on trust function to get accurate data and energy model are utilized to reduce the energy transmission in the WSN.

The remaining section is defined as follows. The Design issues and Related work are discussed in section 2. The LEACH protocol in section 3. The Proposed Protocol is presented in section 4. The Experimental results are performed and contrasted with SEP in section 5. Conclusion and future work describe in section 6 .

2. Design Issues and Related Work. The important task in the sensor network is to enhance the RE of the SN and WSN life by using optimum energy conservation techniques. The WSN area is partitioned into the number of regions by using longitudinal distance to the BS. In [9] the regions constitute $\mathrm{CH}$ for transmitting data to BS via the multihop scheme. In [10] performed static clustering to reduce the overhead in the system. The node having maximum RE is used for the selection of $\mathrm{CH}$ in the WSN. The increased number of $\mathrm{CH}(\mathrm{s})$ in the region enhanced the RE of SN and lifetime of system [11]. In [12] proposed LEACH protocol and performed static clustering for the $\mathrm{CH}$ selection by utilizing maximum RE of SN and minimum distance from BS as parameter in the network. In [13] proposed a minimum spanning tree algorithm for the selection of $\mathrm{CH}$ and transmission of data to BS using a single-hop scheme in the network. In [14] introduced the LEACH protocol for an efficient cluster head selection process and used $\mathrm{K}$ means clustering method for proficient utilization of clusters node inside the network and improved the performance of the WSN. In [15] proposed a clustering technique for the organization of SN and efficient routing scheme for transmission of data to BS. The WSN is partitioned into different regions and $\mathrm{CH}$ is based on the $\mathrm{SN}$ having maximum $\mathrm{RE}$ and minimum distance from BS which enhances the network lifetime. In [16] used distance,energy and node density as a parameter to increase the lifespan of WSN. In [17] energy problem in LEACH protocol is solved by adjusting the CH formula bassed on distance and energy as important parameters in the network. In [18] used radius and weight function to elect candidate $\mathrm{CH}$ in the network area and transmission of data to BS via a multi-hop approach which minimizes the energy consumption in the network. In [19] introduced the K- Medoids algorithm for the selection of clusters inside the network which enhanced the lifespan of the WSN. In [20] introduced a new methodology that uses the fusion method based on the principle of ingredient analysis algorithms to minimize the energy issue in the network. In [21] authors said that unequal nodes energy consumption and $\mathrm{CH}$ selection process is randomly performed in the network. The problem is solved by an efficient CH selection process which enhanced the node's RE and the lifetime of the WSN. In [19] proposed a protocol in which WSN is partitioned into equal size regions and static clustering scheme is used to avoid the overhead problem and multi-hop scheme for transfer the data to the BS. In [22] proposed a protocol in which the $\mathrm{CH}$ and non-CH are selected according to the RE of SN. The maximum value is chosen as $\mathrm{CH}$ among them which reduced the consumption of energy inside the network and improve system performance. The $\mathrm{CH}$ selection in the LEACH protocol is not appropriate so causes various problems.

In [23] introduced trust model for secure data transmission and minimize various issues in the network. In [24] the trust model is used to secure various layers of communication in the network. In [25] proposed data fusion process relying on the degree of trust to enhanced the system performance. In [26] proposed the data fusion method based on multivariate streams of data to detect and avoid outliers in the system. In [27] proposed a Trust-Distrust protocol and use four stages as topology management, linq quality appraisal, grading 
and secure data transmission based on grade points in the network for data routing to the BS. In [28] achieved secure communication based on trust function and energy efficient clustering algorithm in the network. In [29] proposed a protocol for the selection of $\mathrm{CH}$ based on trust function. The data fusion and trust function is utilized to avoid unnecessary transmission and achieve a higher packet delivery ratio in the system.

3. Low Energy Adaptive Cluster Hierarchy (LEACH). The LEACH is the first clustering protocol.The selection of $\mathrm{CH}$ in the protocol is performed randomly and average energy is distributed among all nodes to avoid energy issues and network survival period. In the protocol different clusters are formed and among them, one is elected as $\mathrm{CH}$. The rest non- $\mathrm{CH}(\mathrm{s})$ nodes sent data to each respective $\mathrm{CH}(\mathrm{s})$ to avoid the redundancy problem occurred in the data. The $\mathrm{CH}(\mathrm{s})$ aggregate the data and sent it to $\mathrm{BS}$. The non-CH(s) have the cluster header information and a small routing table is maintained by the CHs [22]. The unnecessary consumption inside the network is avoided by using a routing table in the protocol. The protocol has many advantages as well as disadvantages too. In LEACH protocol when cluster formation takes place, the energy is optimized in the stabilization phase. The random numbers are allocated as 0 and 1 to every SN and when the resulting number is greater compared to the threshold set $\mathrm{T}(\mathrm{n})$ will be chosen as $\mathrm{CH}$ for that round. The clustering process has two phases known as the establishing phase and stabilization phase. The formation of clusters takes place and message-id is broadcast in the network using signal strength and with the help of non- $\mathrm{CH}(\mathrm{s})$ nodes in the network. The $\mathrm{CH}(\mathrm{s})$ request all messages and the routing table is maintained and follow the TDMA schedule for all clusters. The data aggregation is performed on the basis of the routing table and finally, the data is sent to BS [30][31].

4. Proposed Model. The SEP protocol was introduced to minimize energy issues in the network [32][33]. The SEP protocol uses weighted probability for $\mathrm{CH}$ election in normal as well as advance nodes. The SEP protocol does not ensure whether the nodes are effectively deployed or not in the network.

This paper proposes a new protocol in which WSN is partitioned into regions and contains two different SN. The nodes for high energy are known as advanced nodes and for low energy nodes called normal nodes. The proposed protocol is executed in two phases. In the first phase, distribution of SN and new Threshold value for cluster head selection in the WSN. In the next phase the accuracy of data is preserved by utilizing the data fusion method based on trust function to obtained accurate data. The energy model is used to reduce the unnecessary energy transmission in the WSN. The proposed protocol uses new a cluster head selection mechanism which avoids randomness for $\mathrm{CH}$ selection process. The selection of $\mathrm{CH}(\mathrm{s})$ is performed by using maximum RE of SN and minimum BS distance. The new $\mathrm{T}(\mathrm{H})$ consists of distance ratio and weighted energy which avoids energy failure problems in the $\mathrm{CH}(\mathrm{s})$ and low node energy problem. Through this way,it enhances the $\mathrm{CH}(\mathrm{s})$ nodes RE. The $\mathrm{CH}(\mathrm{s})$ wait for the completion of the data communication task of their cluster nodes. The proposed protocol reduces the energy consumption in WSN and improves the system performance.

4.1. Network Assumptions for Designing the Proposed Protocol. The following presumptions are considered for designing the proposed protocol.

- The SN are scattered in a random fashion in the RoI.

- The BS has a continuous power supply and the SN are deterministic in nature.

- The RSSI Plays a major role in the distance estimation between two nodes.

- The battery is not rechargeable.

4.2. Energy Model. The Energy model for the proposed protocol shown in the Fig 4.1. For communicating $c$ bits over distance $d$, the transmission energy $\left(T_{E}\right)$ is required as follows [16]:

$$
T_{E}= \begin{cases}c \times E_{\text {elec }}+c \times E_{f s} \times d^{2}, & \text { if } \mathrm{d}<d_{0} \\ c \times E_{\text {elec }}+c \times E_{m p} \times d^{4}, & \text { else }\end{cases}
$$

where, $E_{\text {elec }}=$ Electrical energy $\left(E_{\text {elec }}\right.$ required for the conversion of 1 bit of data to signal), $E_{f s}=$ Power amount for free space, $E_{m p}=$ Power amount for multipath models.

$$
d_{0}=\sqrt{\frac{E_{f s}}{E_{m p}}}
$$




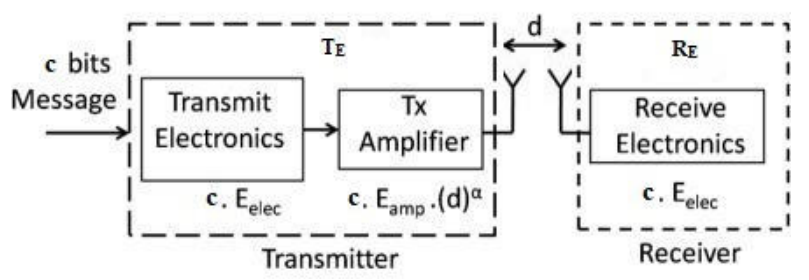

Fig. 4.1: Energy Model

The energy required for receiving $c$ bits is as follows:

$$
E_{R X}=c \times E_{\text {elec }}
$$

The energy required by the Cluster $\operatorname{Member}\left(C M_{E}\right)$ for communicating $c$ bits is as follows:

$$
C M_{E}=c \times E_{\text {elec }}+c \times E_{f s} \times d_{C H}
$$

where $d_{C H}=$ distance to $\mathrm{CH}$.

The energy required by the $\mathrm{CH}\left(\mathrm{CH}_{E}\right)$ is calculated as follows:

$$
C H_{E}=c \times m\left(E_{\text {elec }}+E_{f s} \times d_{B}+E_{D A}\right)
$$

where $m=$ count of cluster member's, $d_{B}=$ distance from Base Station, $E_{D A}=$ Aggregation Energy.

4.3. Trust Function. Let us assume that the set of $\mathrm{SN}$ as $\mathrm{S}=s_{1}, s_{2}, \ldots, s_{n}$ is covering the entire network area. The $d_{i}$ and $d_{j}$ is the data collected by the sensor node $s_{i}$ and $s_{j}$ at same moment. If the accuracy of sensor data $d_{i}$ is higher, than the trust degree of $d_{i}$ is greater than the rest of the sensor data. When data $d_{i}$ is trusted by $d_{j}$, than $d_{i}$ covers all the possible degree for sensor data. Thus the trust degree function is represented as [25]:

$$
t_{i j}=f\left(\left|\left(d_{i}-d_{j}\right)\right|\right)
$$

where $t_{i j}=f\left(\left|\left(d_{i}-d_{j}\right)\right|\right) \in[0,1]$ and $i, j=1,2, \ldots, n$. Based on the trust function $t_{i j}$, the trust matrix corresponding to $n$ number of sensor nodes computes all same parameters used at the same time as follow:

$$
T=\left[\begin{array}{ccc}
t_{11} & \cdots & t_{1 n} \\
\vdots & \ddots & \vdots \\
t_{n 1} & \cdots & t_{n n}
\end{array}\right]
$$

The weight of data $\left(d_{i}\right)$ collected from the sensor node $\left(s_{i}\right)$ is $b_{i}$. In the matrix $T, t_{i j}$ represents the trust degree of $d_{i}$ to $d_{j}$, but does not show the trust degree of all data with respect to $d_{i}$. The actual value of $d_{i}$ is reflected by $t_{i 1}, t_{i 2}, \ldots, t_{i n}$. Therefore, weight matrix $B$ combine all the actual value of $T$ with a set of the non-negative matrix $A$ with values $a_{1}, a_{2}, \ldots, a_{n}$.

$$
\begin{gathered}
{\left[\begin{array}{c}
b_{1} \\
b_{2} \\
\vdots \\
b_{n}
\end{array}\right]=T\left[\begin{array}{c}
a_{1} \\
a_{2} \\
\vdots \\
a_{n}
\end{array}\right]} \\
b_{i}=a_{1} t_{i 1}+a_{2} t_{i 2}+\ldots+a_{n} t_{i n}
\end{gathered}
$$




$$
b_{i}=\sum_{j=1}^{n} a_{j} t_{i j}
$$

The matrix $B$ and $A$ are scalar multiples, if $B=T A=\lambda A$. Here, $\lambda$ is the Eigenvalue for the corresponding eigenvector $A$. Thus the degree of trust is measured as:

$$
\frac{b_{i}}{b_{j}}=\frac{a_{i}}{a_{j}}, i, j=1,2, \ldots, n
$$

The normalized $b_{i}$ is:

$$
b_{i}=\frac{a_{i}}{a_{1}+a_{2}+\ldots+a_{n}}
$$

The data fusion is the process to collects data from multiple sources and gives meaningful information that is not performed by any single sensor node. The objective of data fusion is to enhance the QoS and achieves reliable and accurate data transmission in the RoI. The data fusion process also minimized data redundancy problems and minimized the energy conniption in the WSN. The data fusion expression is:

$$
D_{F}=\sum_{i=1}^{n} b_{i} d_{i}
$$

From equation 4.13 data fusion is also expressed as:

$$
D_{F}=\frac{\sum_{i=1}^{n} a_{i} d_{i}}{a_{1}+a_{2}+\ldots+a_{n}}
$$

4.4. Cluster Head Selection Phase. The $\mathrm{CH}$ are selected by using maximum nodes RE and minimum distance from BS.The nodes are categorized into two parts. The first is normal nodes and the second is advanced nodes.

$$
\begin{gathered}
N_{P}=\frac{P}{1+N_{n} N_{a}} \\
A_{P}=\frac{P}{1+N_{n} N_{a}}\left(1+N_{n}\right)
\end{gathered}
$$

where, $N_{P}=$ Selection probability of normal node as $\mathrm{CH}, A_{P}=$ Selection probability of advanced node as $\mathrm{CH}$, $N_{a}=$ Advanced Node percentage, $N_{n}=$ Amount of energy higher than the normal node.

The threshold is calculated as shown below:

$$
T(H)= \begin{cases}\frac{P}{1-P \times\left(r \bmod \frac{1}{P}\right)}, & \text { if } \mathrm{n} \in \mathrm{G} \\ 0, & \text { otherwise }\end{cases}
$$

where $T(H)=$ Threshold Value, $r=$ current number of round, $P=$ The desired percentage of a node to be CH.

The new $T(H)$ is calculated by modifying in equation 4.17. The weighted energy $(E)$ and distance ratio $(D)$ are calculated as follow:

$$
\begin{gathered}
E=\left(R E_{\text {current }}-\left(E_{a}+E_{t}+E_{r}\right)\right) \\
D=\frac{D_{B S}}{D_{L}}
\end{gathered}
$$

where $R E_{\text {current }}$ is current Residual Energy, $E_{a}$ is Aggregation Energy, $E_{t}$ is Transmission Energy, $E_{r}$ is Reception Energy and $D_{B S}$ is the distance from the BS, $D_{L}$ is the longest distance from BS.The new $T(H)$ is 
used to avoid the low energy problem and improves $\mathrm{CH}$ survival rate. By utilizing equation 4.15, 4.16, 4.17, 4.18, 4.19 simultaneously improves in distribution of Normal and Advanced nodes. The new $T(H)$ for Normal and Advanced nodes are given in equation 4.20 and 4.21 below.

$$
\begin{aligned}
& T(H)= \begin{cases}\frac{N_{P X}}{1-N_{P} \times\left(r \bmod \frac{1}{N_{P}}\right)}(a \times E+b \times D), & \text { if } \mathrm{n} \in \mathrm{G} \\
0, & \text { otherwise }\end{cases} \\
& T(H)= \begin{cases}\frac{A_{P X}}{1-A_{P} \times\left(r \bmod \frac{1}{A_{P}}\right)}(a \times E+b \times D), & \text { if } \mathrm{n} \in \mathrm{G} \\
0, & \text { otherwise }\end{cases}
\end{aligned}
$$

where $N_{P X}$ represents $N_{P}$ with weight parameter X, $A_{P X}$ represents $A_{P}$ with weight parameter $X$, and $a, b$ are the coefficient i.e. $a \in[0,1], b \in[0,1]$ and $a+b=1$.

Equation 4.20 and 4.21 improves energy consumption in the WSN. The long-distance nodes consume much more energy compares to short distance nodes. Therefore by using new $T(H)$ for normal and advanced nodes improves the distribution of SN and enhanced the node RE in the WSN.

4.5. Proposed Protocol. The flow chart for the Proposed protocol is shown in Fig 4.2.

The network consist of advanced nodes and normal nodes. The new threshold value minimizes the randomness in the $\mathrm{CH}$ selection process. The data fusion rate is used to get accurate data using trust function. The energy model are used to avoid unnecessary energy transmission in the network.

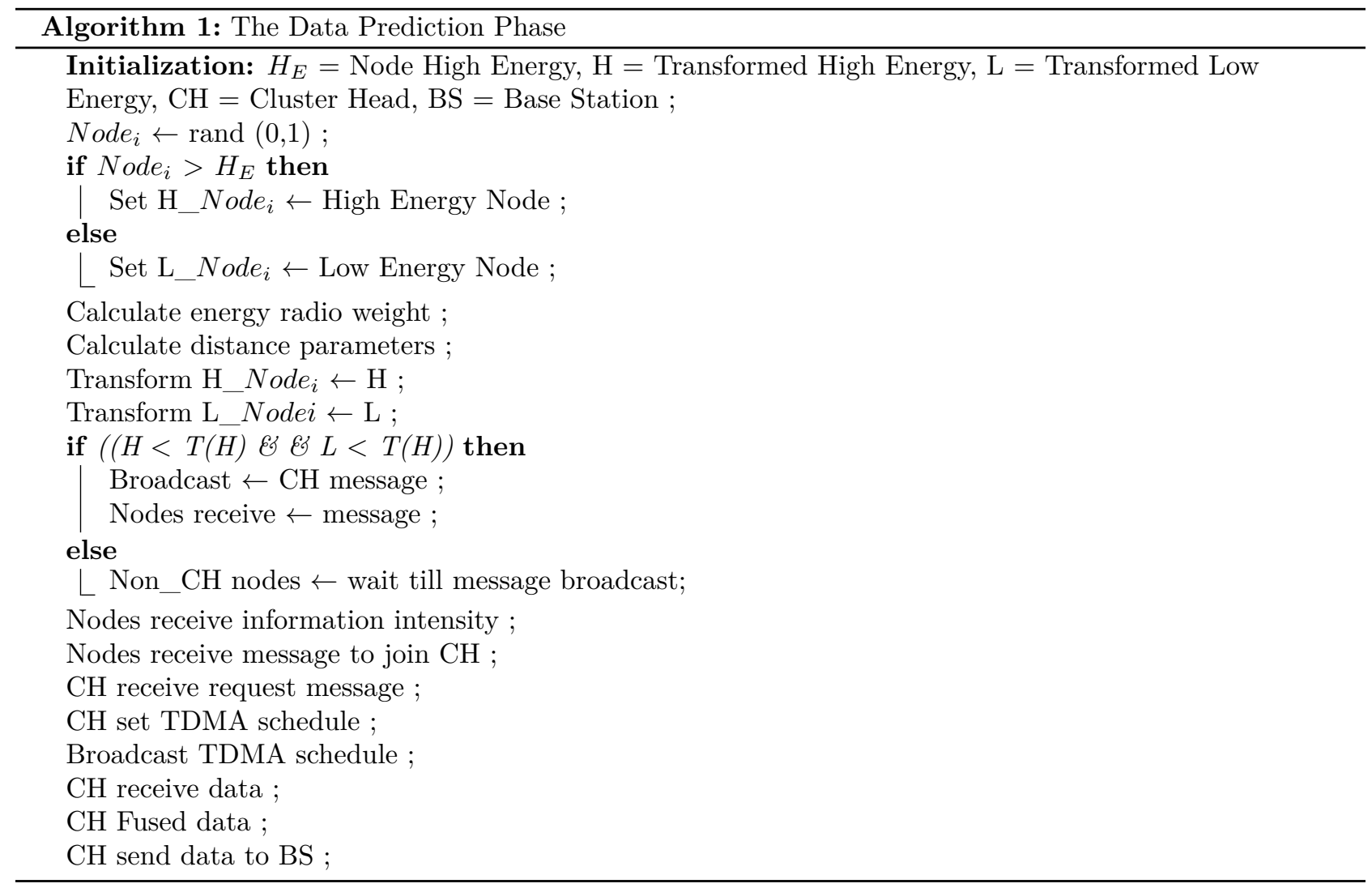




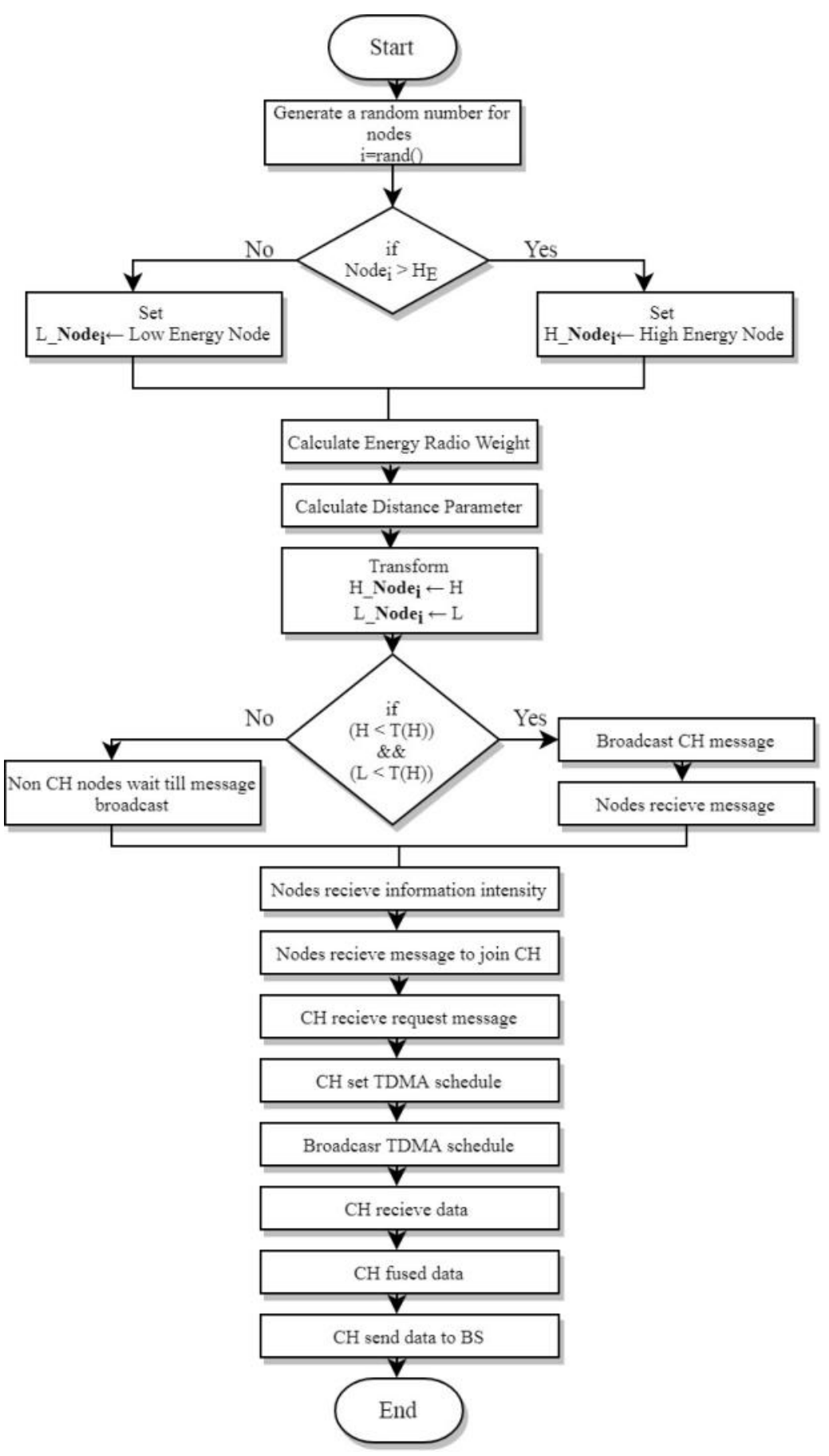

Fig. 4.2: Flow Chart of Proposed Protocol 
Table 5.1: Parameter for Simulation

\begin{tabular}{|l|l|}
\hline Parameters Used & \multicolumn{1}{|c|}{ Values } \\
\hline Nodes (N) & 150 \\
Network Area & $(150,150)$ \\
Position of BS & $(150,75)$ \\
$E_{f s}$ & $10 \mathrm{pJ} / \mathrm{bit} / \mathrm{m}^{2}$ \\
$E_{m p}$ & $0.0013 \mathrm{pJ} / \mathrm{bit} / \mathrm{m}^{4}$ \\
$\left(E_{0}\right)$ & $0.5 \mathrm{~J}$ \\
$\left(E_{R X}\right)$ & $50 \mathrm{~nJ} / \mathrm{bit}$ \\
$\left(E_{D A}\right)$ & $5 \mathrm{~nJ} / \mathrm{bit} /$ signal \\
\hline
\end{tabular}

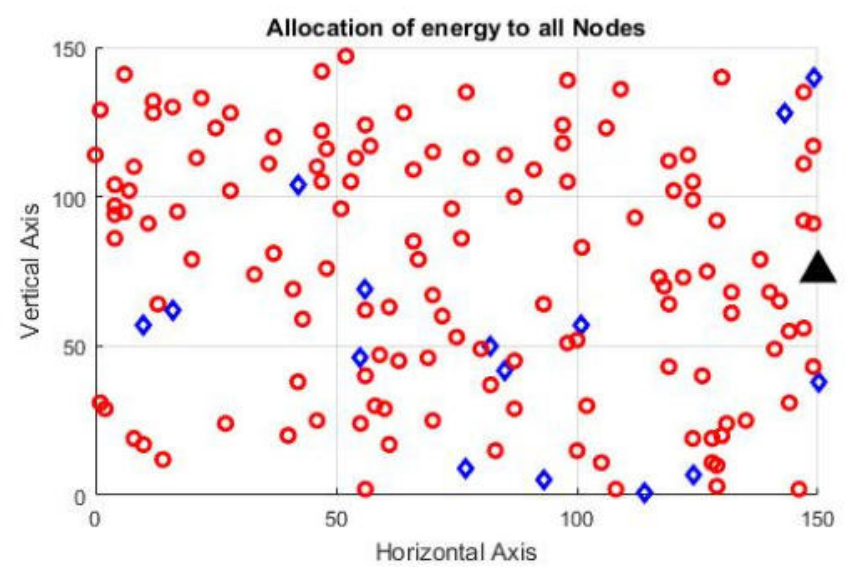

Fig. 5.1: The deployment of Normal Nodes and Advanced Nodes

5. Simulation Results And Execution Assessments. The simulation is performed in Matlab 2017a. The RoI is $(150 \times 150) \mathrm{m}^{2}$ area and the distribution of nodes are deterministic in the area. The probability of $\mathrm{CH}$ selection is $10 \%$. The proposed protocol is compared with the SEP based on simulation parameter [34] as shown in Table 5.1.

The initial network deployment is as shown in the Figure 5.1.

In the Figure 5.1 the blue color represents the advanced nodes and the red color represents the normal nodes. The BS is located at $(150,75)$ on the $X$ axis and the $Y$ axis respectively and represented with black color. The effectiveness of the proposed protocol is compared with respect to the SEP for alive nodes is shown in Figure 5.2, dead nodes in Figure 5.3, number of packet transmitted to BS in Figure 5.4, nodes elected as CH in Figure 5.5, percentage of energy consumed in Figure 5.6, percentage of remaining energy in Figure 5.7 and survival rate percentage in Figure 5.8 respectively.

The results in Figure 5.2 shown that the proposed protocol has 29 alive nodes after the first iteration which is better than SEP.

In the Figure 5.3 shown the proposed protocol reveals better performance than SEP protocol because in the proposed protocol first node dies at 998 rounds where as in SEP protocol dies at 411 rounds.

As shown in the Figure 5.4, the packets transmitted to BS are $1.8 \times 10^{4}$ for the proposed protocol while in SEP protocol the value $1.7 \times 10^{4}$ has used for packet transmission.

As shown in Figure 5.5, the proposed protocol has more number of nodes as $\mathrm{CH}$ compares to the SEP protocol. It can be observed from the figure that the number of $\mathrm{CH}$ starts decreasing after 400 rounds in SEP and for the proposed protocol number of $\mathrm{CH}$ starts decreasing after 1000 rounds. So we can say that the stability period is higher for the proposed protocol compared with SEP.

In the Figure 5.6 shown that the energy consumption is reduced for the proposed protocol compared with 


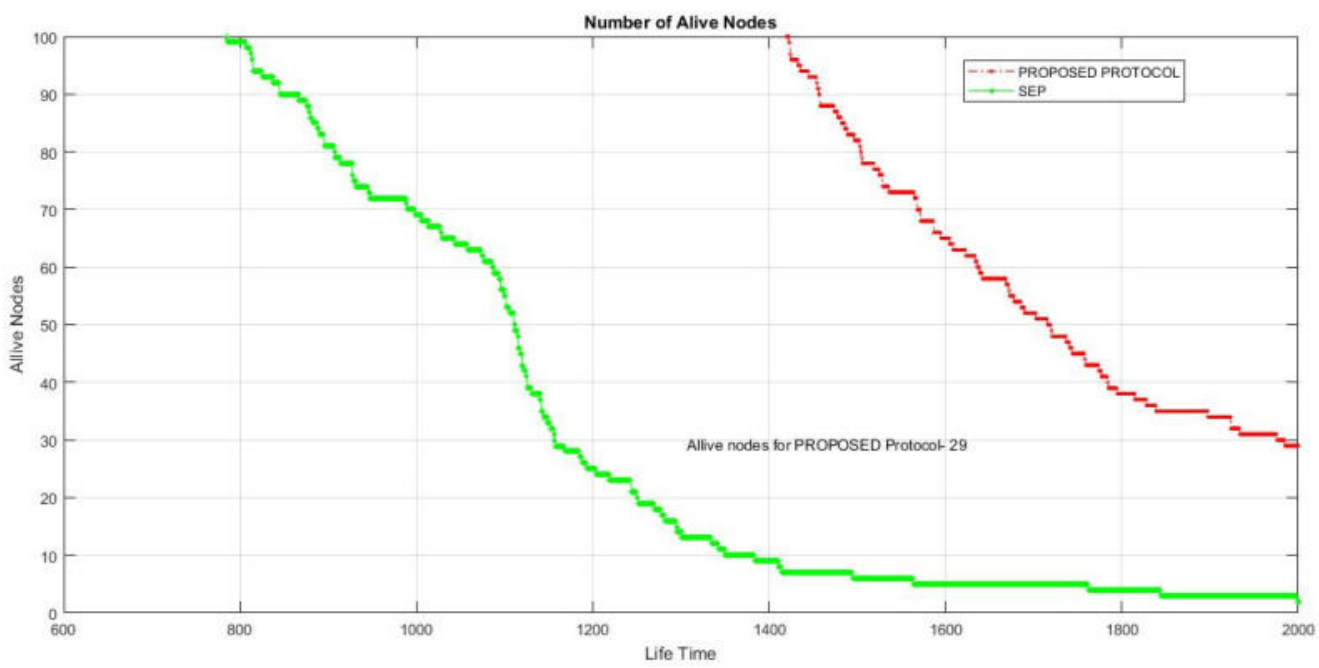

Fig. 5.2: Number of Alive Nodes

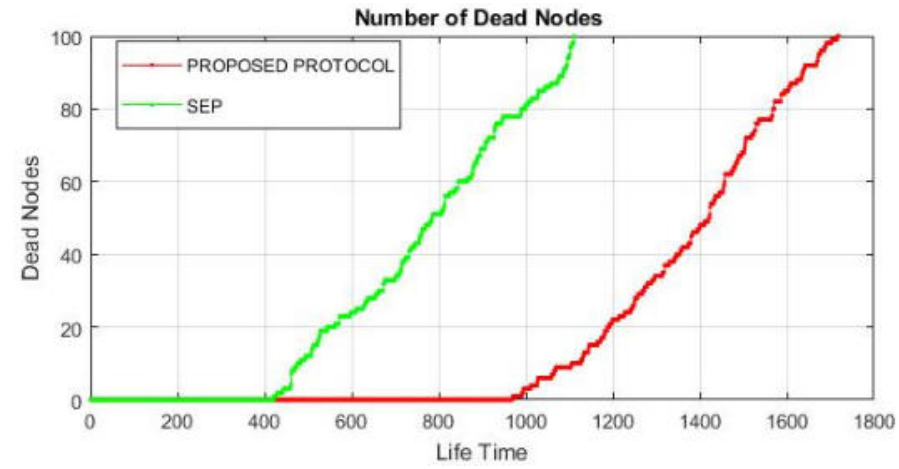

Fig. 5.3: Number of Dead Nodes

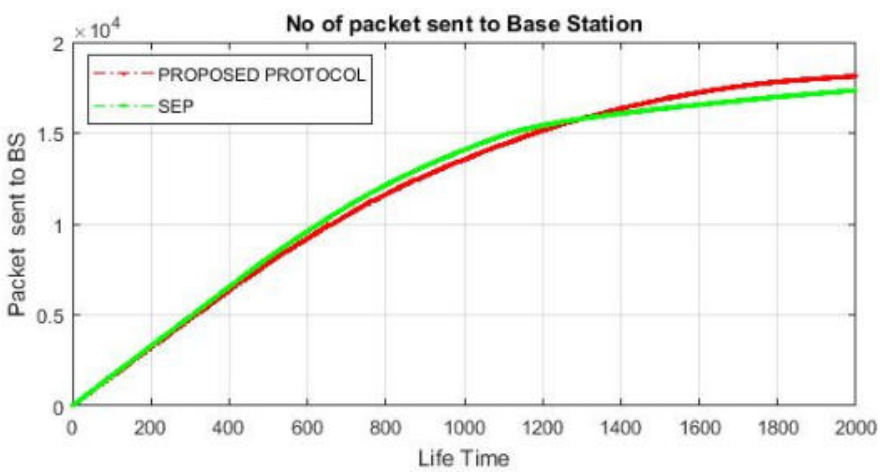

Fig. 5.4: Number of Packet Transmission to BS 


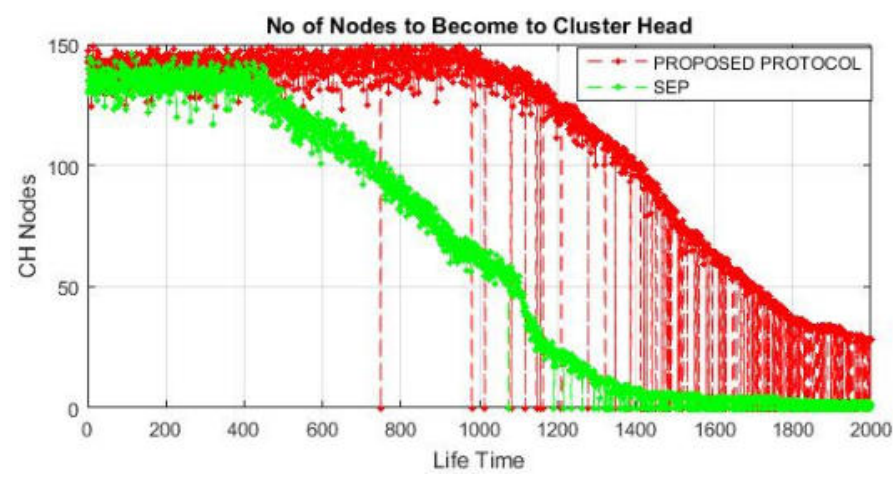

Fig. 5.5: Number of Node to become $\mathrm{CH}$

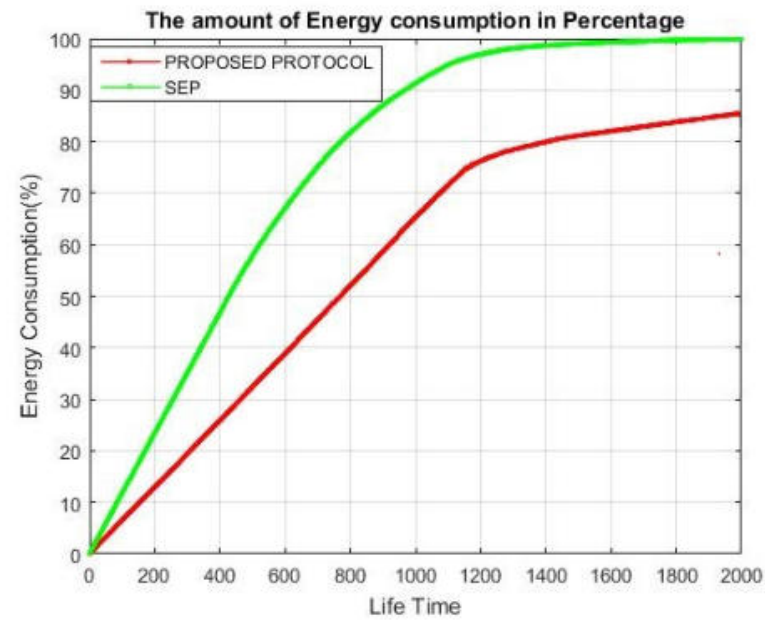

Fig. 5.6: The amount of energy consumption (in \%)

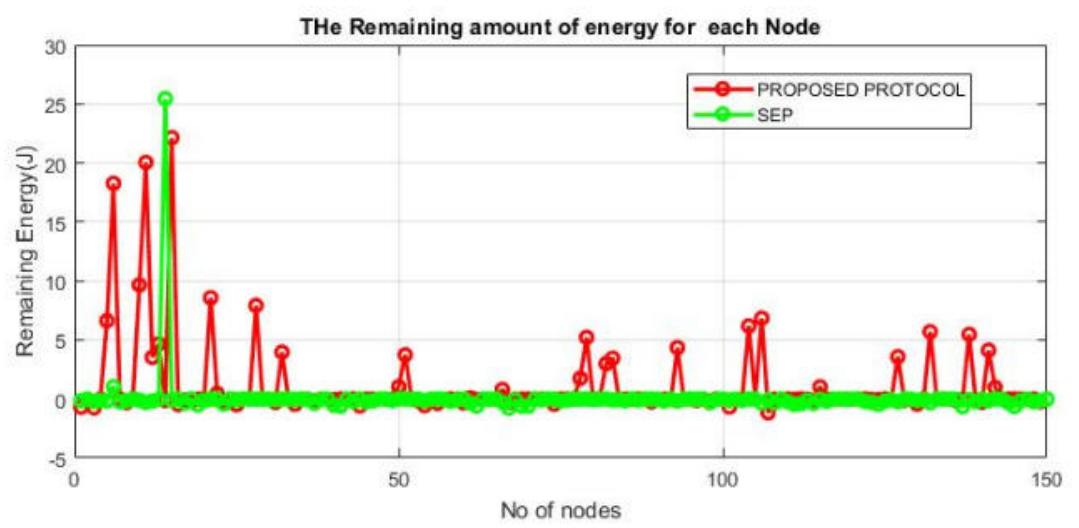

Fig. 5.7: The remaining amount of energy 


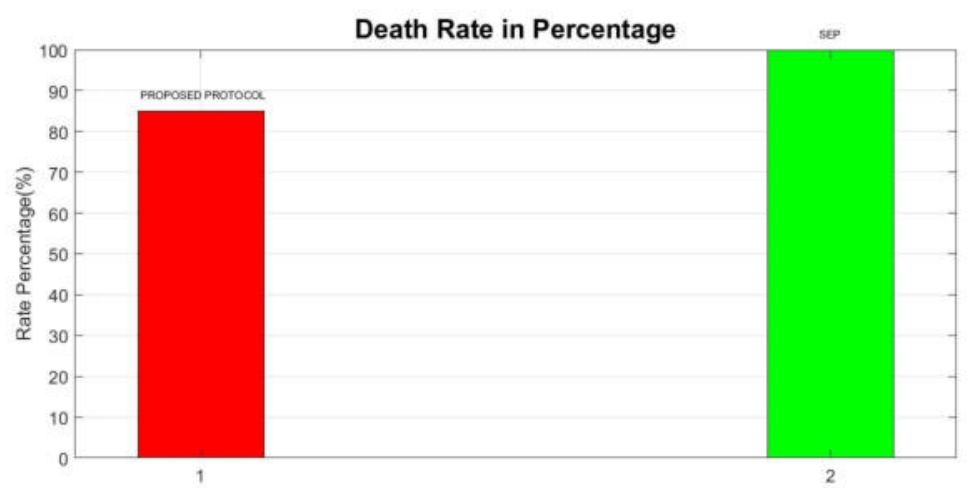

Fig. 5.8: The Death Rate of Network

SEP protocol. It is observed that the energy consumption is around $100 \%$ in SEP protocol for 1400 rounds whereas it is around $80 \%$ for 1400 rounds in the proposed protocol.

In the Figure 5.7 the proposed protocol has more remaining energy compare to the SEP protocol which has almost zero energy for each node.

In the Figure 5.8 the survival rate of the proposed protocol is around $15 \%$ which is significantly better in comparison with SEP.

6. Conclusions. The proposed protocol prevents randomness in the selection process of $\mathrm{CH}$. The new Threshold value consists of weighted energy and distance ratio which prevent the energy issues inside the sensor network. The data fusion method is used in the Proposed protocol to get accurate data using the trust function and the energy model is utilized to minimize energy trnasmission within the network. The simulation results have shown a better lifetime, stability period and survival rate for the Proposed protocol compared with the SEP protocol. In the future fuzzy logic approach will be used for the $\mathrm{CH}$ selection process which efficiently utilized energy consumption inside the network and enhanced the system performance.

\section{REFERENCES}

[1] Munir, Saad Ahmed and Ren, Biao and Jiao, Weiwei and Wang, Bin and Xie, Dongliang and Ma, Jian. Mobile wireless sensor network: Architecture and enabling technologies for ubiquitous computing. Cluster Computing, 2(3), pp. 113-120,(2007). Springer.

[2] V. NArayan, A. Daniel, Novel protocol for detection and optimization of overlapping coverage in wireless sensor networks (2019).

[3] Roy, Nihar Ranjan and Chandra, Pravin. Analysis of data aggregation techniques in wsn. Cluster Computing, 22(3), pp. 571-581,(2020). Springer.

[4] Narayan, Vipul and Daniel, AK and Rai, Ashok Kumar. Energy Efficient Two Tier Cluster Based Protocol for Wireless Sensor Network. Cluster Computing, 22(3), pp. 574-579,(2020). Springer.'

[5] Chaturvedi, Pooja and Daniel, AK. Trust based node scheduling protocol for target coverage in wireless sensor networks. Cluster Computing, 22(3), pp. 163-173,(2015). Springer.

[6] Chaturvedi, Pooja and Daniel, AK. Trust based energy efficient coverage preserving protocol for wireless sensor networks. Cluster Computing, 22(3), pp. 860-865,(2015). Springer.

[7] Chaturvedi, Pooja and Daniel, Ajai K. Trust Based Target Coverage Protocol for Wireless Sensor Networks Using Fuzzy Logic. Cluster Computing, 22(3), pp. 188-192,(2016). Springer.

[8] Tripathi, Abhishek and Gupta, Hari Prabhat and Dutta, Tanima and Mishra, Rahul and Shukla, KK and Jit, SATYABRat. Coverage and connectivity in WSNs: A survey, research issues and challenges. IEEE Access, 6(3), pp. 26971-26992,(2018). IEEE.

[9] Rajpoot, Prince and Dwivedi, Pragya. Optimized and load balanced clustering for wireless sensor networks to increase the lifetime of WSN using MADM approaches. Wireless Networks, 26(1), pp. 215-251,(2020). Springer.

[10] Yadav, Ravi and Daniel, AK. Fuzzy based smart farming using wireless sensor network. Cluster Computing, 22(3), pp. 1-6,(2018). Springer. 
[11] Lu, Yu-ding and Chen, Yao-dong and Chen, Meng-Yuan. The improvement and simulation research of wireless sensor network LEACHprotocol. Journal of Anhui Polytechnic University, 22(4), pp. 13,(2012). Springer.

[12] Shokouhifar, Mohammad and Jalali, Ali. A new evolutionary based application specific routing protocol for clustered wireless sensor networks. AEU-International Journal of Electronics and Communications, 69(1), pp. 432-441,(2015). Elsevier.

[13] Zhenxing, Wang and Weili, Xiong and Baoguo, Xu. A LeACH Cluster Tree Network Routing Algorithm Research [J]. Computer Measurement \& Control, 11(3), pp. 5811-5823,(2008). Springer.

[14] Jiang, JianMing and Shi, GuoDong and Zhao, Dean and Li, ZhengMing and Shi, Bing and Zhao, Yigang et al. Intelligent monitoring system of aquaculture parameters based on $\mathrm{LEACH}$ protocol.. Nongye Jixie Xuebao= Transactions of the Chinese Society for Agricultural Machinery, 45(11), pp. 286-291,(2014). Chinese Society for Agricultural Machinery.

[15] Li, Fangfang And Wang, Jing. A New LEACH-Based Routing Algorithm for Wireless Sensor Networks [J]. Chinese Journal of Sensors and Actuators, 10(3), pp. 5811-5823,(2012). Springer.

[16] Wan, Chuanfei and Du, Shangfeng. Improvement and simulation of leach in wireless sensor networks. Jisuanji Yingyong yu Ruanjian, 28(4), pp. 113-116,(2011). Shanghai Institute of Computing Technology.

[17] Roshan, Komal and Sharma, Kritika Rai. Improved LEACH protocol with cache nodes to increase lifetime of wireless sensor networks. Cluster Computing, 22(3), pp. 903-908,(2018). Springer.

[18] Zhang, Li. The improvement and simulation of LEACH clustering routing protocol for WSNs. Wuhan University of Technology, Wuhan, 22(3), pp. 1-75,(2009). Springer.

[19] Zayoud, Maha and Abdulsalam, Hanady M and Al-Yatama, A and Kadry, Seifedine. Split and merge leach based Routing algorithm for wireless sensor networks. International Journal of Communication Networks and Information Security, 10(1), pp. 155-162,(2018). Springer.

[20] Xu, Yan and Yue, Zhanwei and Lv, Lingling. Clustering routing algorithm and simulation of internet of things perception layer based on energy balance. IEEE Access, 7(3), pp. 145667-145676,(2019). IEEE.

[21] Gawade, Rohit D and Nalbalwar, Sanjay L. A centralized energy efficient distance based routing protocol for wireless sensor networks. Journal of Sensors, 2016(3), pp. 5811-5823,(2016). Hindawi.

[22] Wu, Wenliang and Xiong, Naixue and Wu, Chunxue. Improved clustering algorithm based on energy consumption in wireless sensor networks. Iet Networks, 6(3), pp. 47-53,(2017). IET.

[23] Dhand, GeEtika And Tyagi, SS. SMEER: Secure multi-tier energy efficient routing protocol for hierarchical wireless sensor networks. Wireless Personal Communications, 105(1), pp. 17-35,(2019). Springer.'

[24] Dwivedi, AK and Sharma, AK and Kumar, R. Dynamic Trust Management Model for the Internet of Things and Smart Sensors: The Challenges and Applications. Recent Patents Comput. Sci, 12(3), pp. 5811-5823,(2019). Springer.

[25] Sun, Guiling and Zhang, Ziyang and Zheng, Bowen and Li, Yangyang. Multi-Sensor Data Fusion Algorithm Based on Trust Degree and Improved Genetics. Sensors, 19(9), pp. 2139,(2019). Multidisciplinary Digital Publishing Institute.

[26] Kolomvatsos, Kostas and Anagnostopoulos, Christos and Hadjiefthymiades, Stathes. Data fusion and type-2 fuzzy inference in contextual data stream monitoring. IEEE Transactions on Systems, Man, and Cybernetics: Systems, 47(8), pp. 1839-1853,(2016). IEEE.

[27] KarThick, SuYAmbu. TDP: A novel secure and energy aware routing protocol for wireless sensor networks. International Journal of Intelligent Engineering and Systems, 11(2), pp. 76-84,(2018). Springer.

[28] Sharma, Richa and Vashisht, Vasudha and Singh, Umang. eeTMFO/GA: a secure and energy efficient cluster head selection in wireless sensor networks. Telecommunication Systems, 22(3), pp. 1-16,(2020). Springer.

[29] Mishra, Mukesh and Gupta, Gourab Sen and Gui, Xiang. Trust-Based Cluster Head Selection Using the K-Means Algorithm for Wireless Sensor Networks. Cluster Computing, 22(3), pp. 819-825,(2019). Springer.

[30] Al-Humidi, Nada and Chowdhary, GiRish V. Energy-aware approach for routing protocol by using centralized control clustering algorithm in wireless sensor networks. Cluster Computing, 22(3), pp. 261-274,(2019). Springer.

[31] Gopalakrishna, Aravind Kota and Pai, Manohara MM. Multi-service adaptable routing protocol for wireless sensor networks. Cluster Computing, 22(3), pp. 5811-5823,(oct " 23" 2012). Google Patents.

[32] Heinzelman, Wendi Beth. Application-specific protocol architectures for wireless networks. Cluster Computing, 22(3), pp. $5811-5823,(2000)$. Springer.

[33] Smaragdakis, Georgios and Matta, Ibrahim and Bestavros, Azer. SeP: A stable election protocol for clustered heterogeneous wireless sensor networks. Cluster Computing, 22(3), pp. 5811-5823,(2004). Springer.

[34] Dwivedi, Anshu Kumar and Sharma, Awadhesh Kumar and Mehra, Pawan Singh. Energy Efficient Sensor Node Deployment Scheme for Two Stage Routing Protocol of Wireless Sensor Networks assisted IoT. ECTI Transactions on Electrical Engineering, Electronics, and Communications, 18(2), pp. 158-169,(2020). IEEE.'

Edited by: Dana Petcu

Received: Sep 30, 2020

Accepted: Jan 3, 2021 